\title{
Investigation of Quasi-periodic Variations in Hard $X$-Rays of Solar Flares. II. Further Investigation of Oscillating Magnetic Traps
}

\author{
J. Jakimiec • M. Tomczak
}

Received: 23 September 2010 / Accepted: 4 January 2012 / Published online: 3 February 2012

(C) The Author(s) 2012. This article is published with open access at Springerlink.com

\begin{abstract}
In our recent paper (Jakimiec and Tomczak, Solar Physics 261, 233, 2010) we investigated quasi-periodic oscillations of hard X-rays during the impulsive phase of solar flares. We have come to the conclusion that they are caused by magnetosonic oscillations of magnetic traps within the volume of hard-X-ray (HXR) loop-top sources. In the present paper we investigate four flares that show clear quasi-periodic sequences of the HXR pulses. We also describe our phenomenological model of oscillating magnetic traps to show that it can explain the observed properties of the HXR oscillations. The main results are the following: i) Low-amplitude quasi-periodic oscillations occur before the impulsive phase of some flares. ii) The quasi-periodicity of the oscillations can change in some flares. We interpret this as being due to changes of the length of oscillating magnetic traps. iii) During the impulsive phase a significant part of the energy of accelerated (non-thermal) electrons is deposited within a HXR loop-top source. iv) The quick development of the impulsive phase is due to feedback between the pressure pulses by accelerated electrons and the amplitude of the magnetic-trap oscillation. v) The electron number density and magnetic field strength values obtained for the HXR loop-top sources in several flares fall within the limits of $N \approx$ $(2-15) \times 10^{10} \mathrm{~cm}^{-3}, B \approx(45-130)$ gauss. These results show that the HXR quasi-periodic oscillations contain important information about the energy release in solar flares.
\end{abstract}

Keywords Flares $\cdot$ Energetic particles $\cdot$ Impulsive phase $\cdot$ Oscillations

\section{Introduction}

In hard X-ray (HXR) emission of many flares quasi-periodic variations have been observed with time intervals between the pulses $P \sim 10-60$ s (see Lipa, 1978; see also the review of Nakariakov and Melnikov, 2009 and references therein).

J. Jakimiec $\cdot$ M. Tomczak ( $\varangle)$

Astronomical Institute, University of Wrocław, ul. Kopernika 11, 51-622 Wrocław, Poland

e-mail: tomczak@astro.uni.wroc.pl

J. Jakimiec

e-mail: jjakim @astro.uni.wroc.pl 
In our previous paper (Jakimiec and Tomczak, 2010, Paper I) we attempted to investigate the relationship between the sizes of HXR loop-top sources and quasi-periodic variations. The main difficulty was that sequences of pulses were usually short, so that it is difficult to carry out a comprehensive analysis of their quasi-periodicity. Therefore, we used the mean time interval, $\Delta t$, between the strongest pulses as a simple estimate of the quasiperiodicity $P$.

In the present paper we have selected four flares that have longer sequences of the HXR pulses, so that it was possible to carry out a detailed analysis of their quasi-periodicity (Section 2.1). Section 2.2 contains a detailed analysis of the 16 January 1994 flare. In Section 2.3 we estimate values of the electron density and magnetic field strength inside several HXR loop-top sources. Section 3 contains a discussion and our conclusions.

\section{Observations and Their Analysis}

We used HXR observations recorded by the Yohkoh Hard X-ray Telescope, HXT, (Kosugi et al., 1991) [light curves and images] and the Compton Gamma Ray Observatory Burst and Transient Source Experiment, BATSE (Fishman et al., 1992) [light curves].

\subsection{Analysis of Quasi-periodicity of the HXR Pulses}

We have selected four flares which have longer sequences of the HXR pulses, so that a detailed analysis of their quasi-periodicity was possible. The main difficulty in the analysis was the fact that during the impulsive phase the pulses occurred simultaneously with a quick increase of the total HXR intensity (see Figure 1a). Therefore, we have applied a method of normalization that is commonly used by radioastronomers (see Fleishman, Bastian, and Gary, 2008), to separate HXR pulses from the impulsive phase rise. The normalized time series, $S(t)$, is

$$
S(t)=\frac{F(t)-\hat{F}(t)}{\hat{F}(t)},
$$

where $F(t)$ is the measured HXR flux and $\hat{F}(t)$ is the running average of $F(t)$. The red line in Figure 1a shows $\hat{F}(t)$ calculated with averaging time $\delta t=30 \mathrm{~s}$. The normalized time series, $S(t)$, is shown in Figure 1b. Our basic method to recognize quasi-periodic sequences is the following. We measure the time intervals, $P_{i}$, between successive HXR peaks and calculate the period, $P=\left\langle P_{i}\right\rangle$, and its standard (r.m.s.) deviation, $\sigma(P)$. Our criterion of quasi-periodicity is $\sigma(P) / P \ll 1$. The values of $P$ and $\sigma(P)$ for different parts of the impulsive phase of the investigated flares are given in Table 1 . We interpret the random variations of $P_{i}$ as being due to variations of the length of magnetic traps and of the Alfvén speed inside the traps (see Section 3).

The advantage of $S(t)$ time series is that it allows one to investigate time profiles of HXR pulses (see panels b in Figures 1, 3, 5, 6, 8, and 9). Typically, profiles are symmetrical (quasisinusoidal) and their width is preserved during a sequence (exceptions are very sharp peaks, like that at 22:04:40 UT in Figure 1). This quasi-sinusoidal shape of the pulses supports the idea that they are due to eigen-oscillations of the magnetic structure (traps).

Some authors (e.g. Fleishman, Bastian, and Gary, 2008; Melnikov et al., 2005) used the Fourier transform ("power spectrum") of $S(t)$ time series to determine the mean time interval, $P$, between the successive peaks. Therefore, we have also calculated the power spectra of $S(t)$ (see panels c and d in Figures 1, 3, 5, 6, 8, and 9) in order to compare 

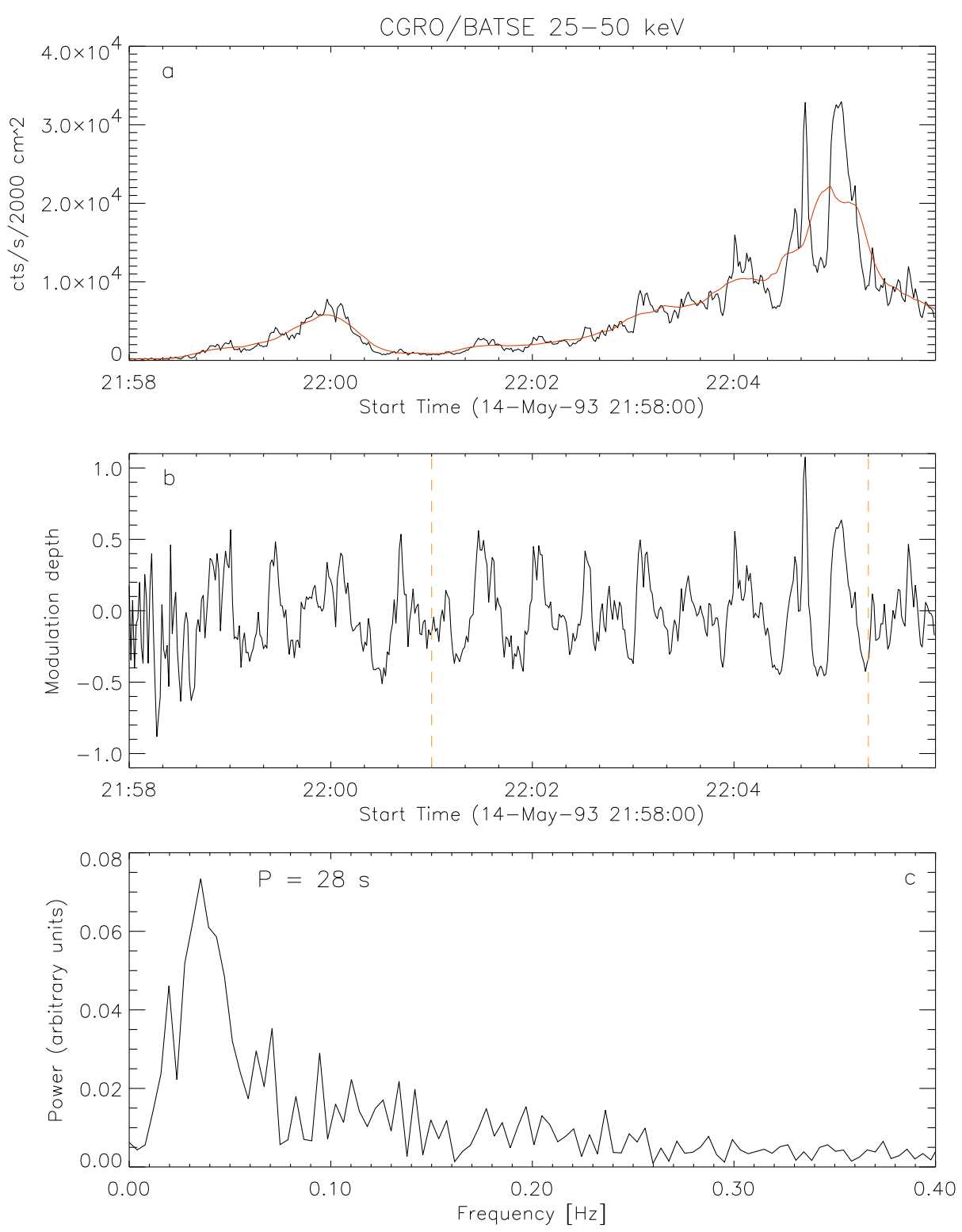

Figure 1 The analysis of the HXR light curve (25-50 keV, Compton Gamma Ray Observatory/BATSE observations) for the flare of 14 May 1993. (a) HXR light curve. The red line shows running average calculated with averaging time $\delta t=30 \mathrm{~s}$. (b) Normalized light curve, $S(t)$ [see Equation (1)]. Vertical dashed lines show the time interval (the impulsive phase) that was used to calculate the power spectrum. (c) The power spectrum calculated for the normalized light curve, $S(t) . P$ is the period corresponding to the peak in the power spectrum.

the obtained values of $P$ with our values given in Table 1 . We see that the values of $P$ obtained (i.e. the main peaks in the power spectra) are in good agreement with our values. 
Table 1 Parameters of the quasi-periodic oscillations.

\begin{tabular}{|c|c|c|c|c|c|c|c|c|c|}
\hline \multirow[t]{2}{*}{ No. } & \multirow[t]{2}{*}{ Date, UT } & \multicolumn{2}{|c|}{$\begin{array}{l}\text { Before } \\
\text { impulsive } \\
\text { phase }\end{array}$} & \multicolumn{2}{|c|}{$\begin{array}{l}\text { During } \\
\text { impulsive } \\
\text { phase rise }\end{array}$} & \multicolumn{2}{|c|}{$\begin{array}{l}\text { At impulsive } \\
\text { phase } \\
\text { maximum }\end{array}$} & \multicolumn{2}{|c|}{$\begin{array}{l}\text { After } \\
\text { impulsive } \\
\text { phase }\end{array}$} \\
\hline & & $P[\mathrm{sec}]$ & Amp $S$ & $P[\mathrm{sec}]$ & $\operatorname{Amp} S$ & $P[\mathrm{sec}]$ & Amp $S$ & $P[\mathrm{sec}]$ & Amp $S$ \\
\hline 1 & $\begin{array}{l}14 \text { May } 1993 \\
21: 58-22: 06\end{array}$ & $32 \pm 4$ & 0.62 & $31 \pm 2$ & 0.70 & 23 & 1.15 & - & - \\
\hline 2 & $\begin{array}{l}\text { 16 January } 1994 \\
23: 08-23: 24\end{array}$ & $47 \pm 4$ & 0.47 & $30 \pm 3$ & 0.28 & $47 \pm 0$ & 0.57 & $43 \pm 3$ & 0.20 \\
\hline 3 & $\begin{array}{l}18 \text { August } 1998 \\
08: 18-08: 23\end{array}$ & - & - & $15 \pm 1$ & 0.26 & $27 \pm 1$ & 0.28 & $28 \pm 3$ & 0.27 \\
\hline 4 & $\begin{array}{l}12 \text { March } 1993 \\
17: 52-18: 04\end{array}$ & $56 \pm 5$ & 0.30 & $16 \pm 1$ & 0.18 & 26 & 0.30 & $18 \pm 2$ & 0.33 \\
\hline
\end{tabular}

$P$ is the mean time interval between successive HXR peaks, Amp $S$ is the mean value of full amplitude of the $S$-function measured for individual HXR peaks.

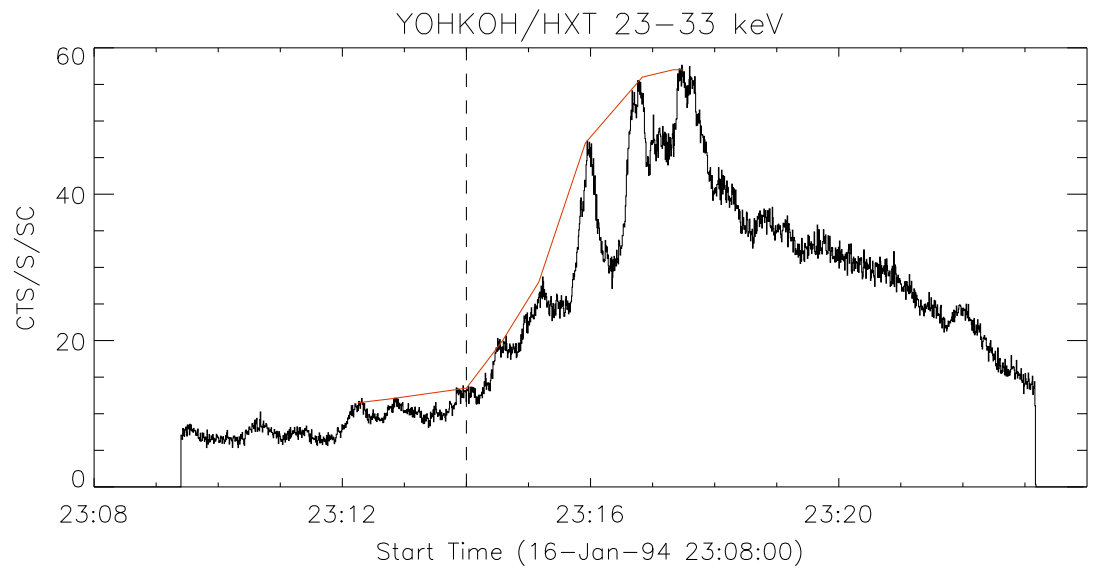

Figure 2 23-33 keV light curve recorded by the Yohkoh/Hard X-ray Telescope for the flare of 16 January 1994. The red line connects peaks of HXR pulses to visualize their non-linear increase and saturation. The noise is due to statistical fluctuations of the counting rate. The dashed vertical line shows the beginning of the impulsive phase.

We see in Figures $1 \mathrm{a}$ and $1 \mathrm{~b}$ that before the impulsive phase, between 21:58:40 and 22:00:20 UT, three increasing pulses occurred. The mean time interval between the pulses is about $31 \mathrm{~s}$, which is close to the quasi-periodicity, $P$, seen in the power spectrum $(28 \mathrm{~s})$ and in Table 1 (32 s). This suggests that these pulses also belong to the quasi-periodic sequence seen during the impulsive phase.

Figure 2 shows the HXR light curve of the 16 January 1994 flare. Like in Figure 1a, during the impulsive phase we see a sequence of increasing pulses. The red line connects the tops of the pulses to show their quick non-linear increase and saturation. Before the impulsive phase there is a sequence of weak pulses. The normalization and power-spectrum analysis is shown in Figure 3. The power spectrum in Figure 3c has been calculated for time interval $\mathrm{A}$, i.e. before and during the impulsive phase. Figure $3 \mathrm{~d}$ shows the power spectrum 

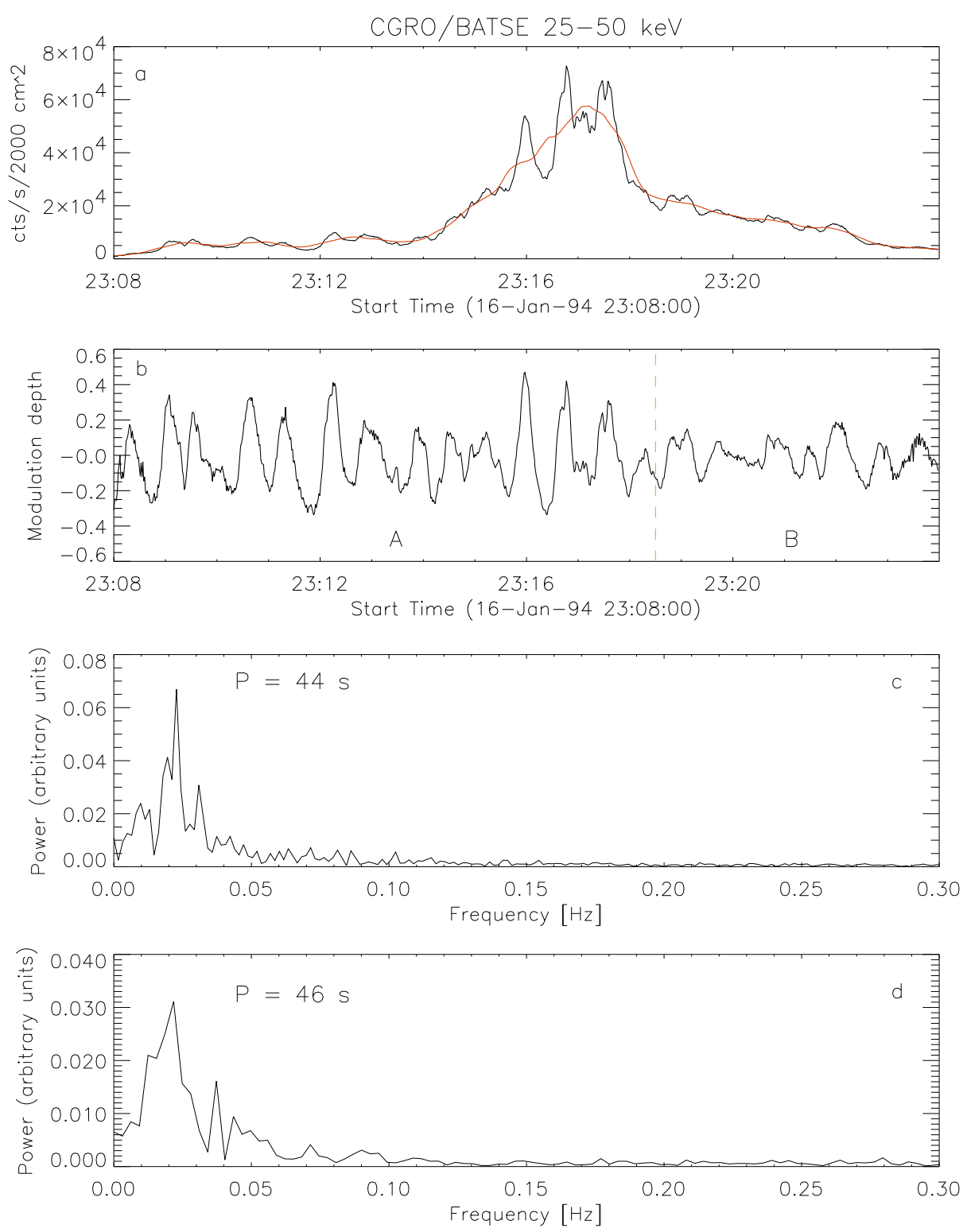

Figure 3 The same as in Figure 1, for the flare of 16 January 1994. The power spectra have been calculated for time intervals A and B and they are shown in panels (c) and (d), respectively.

calculated for the decay phase (time interval B), where the pulses were weak and their profiles were disturbed, but the quasi-period had been retained.

Figure 4 shows the HXR light curve of the 18 August 1998 flare. Its characteristic feature is that quasi-periodicity during the increase of HXR emission is shorter than during its decrease of the emission. Therefore analysis of the light curve has been done separately for time intervals A and B (see Figures 5 and 6). In our model of oscillating magnetic traps 


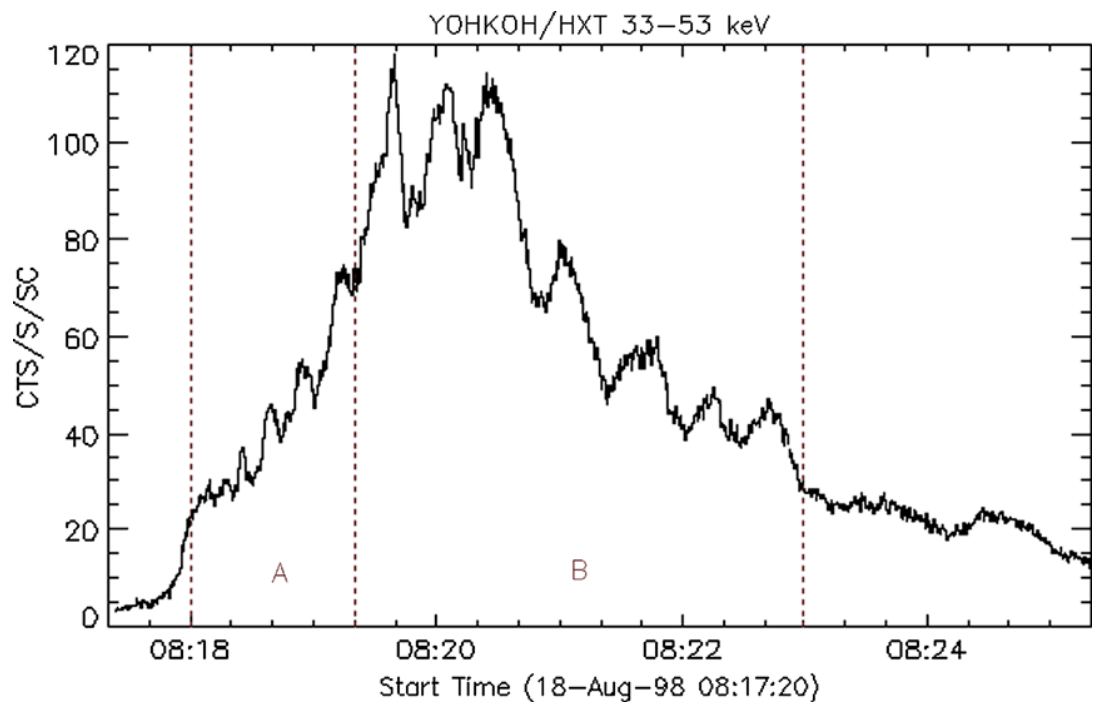

Figure $433-53 \mathrm{keV}$ Yohkoh/HXT light curve for the flare of 18 August 1998. The power spectrum analysis has been carried out for time intervals A and B, separately, and the results are presented in Figures 5 and 6.

changes of quasi-periodicity are explained as being due to changes of the length of the oscillating traps (see Section 3).

Figure 7 shows the HXR light curves of the 12 March 1993 flare. The general behavior is similar to that seen in Figure 2: There are low-amplitude oscillations before the impulsive phase and a quick increase of the HXR emission after the onset of the impulsive phase. The specific features are short-period oscillations after the HXR maximum. Therefore, an analysis of the light curve has been done separately for the time intervals A and B (see Figures 8 and 9). The change of the quasi-periodicity is clearly seen.

The characteristic features of the HXR light curves are the following.

i) There are two components of the HXR emission: sequences of the quasi-periodic pulses and a "quasi-smooth" component (emission below the pulses in Figures 1-9).

ii) Both these components of the HXR emission increase simultaneously during the impulsive phase rise.

The full amplitude, Amp $S$, of the S-function for a HXR pulse is a measure of the ratio of the pulse intensity to the quasi-smooth component; mean values of Amp $S$ are given in Table 1. The results obtained are discussed in Section 3.

\subsection{Analysis of the 16 January 1994 Flare}

We have chosen the flare on 16 January 1994 for further analysis because loop-top and footpoint sources can easily be recognized in its HXR images (Figure 10). We wanted to investigate what had happened in the loop-top source during the transition from low-amplitude oscillations to the quick increase of the HXR emission seen in Figures 2 and 3. Toward this end we have reconstructed the Yohkoh/HXR images in the energy channels L (14-23 keV), M1 (23-33 keV), and M2 (33-53 keV) for the time before impulsive phase oscillations (the upper row in Figure 10), at about the beginning of the impulsive phase (the middle row) and for the maximum of the impulsive phase (the lower row). 

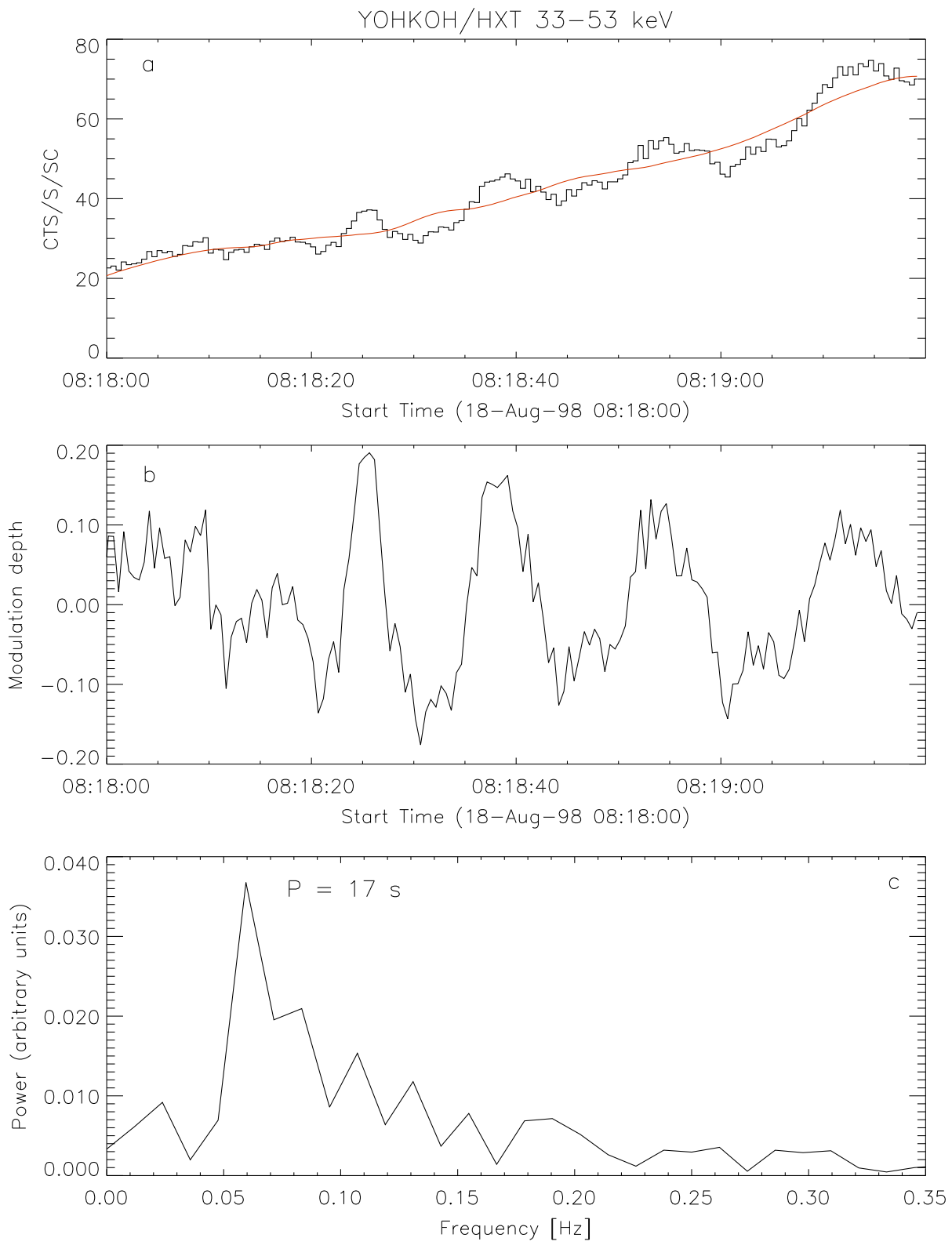

Figure 5 The power-spectrum analysis for the time interval A of the flare on 18 August 1998 (cf. Figure 4).

We see that before the impulsive phase the HXR footpoints are strong, which means that accelerated electrons easily escape from the loop-top source. But at the beginning of the impulsive phase the footpoints are weaker than the loop-top source in the 14-23 and 23$33 \mathrm{keV}$ channels emission, which indicates that most of the accelerated electrons deposit their energy within the loop-top source. 

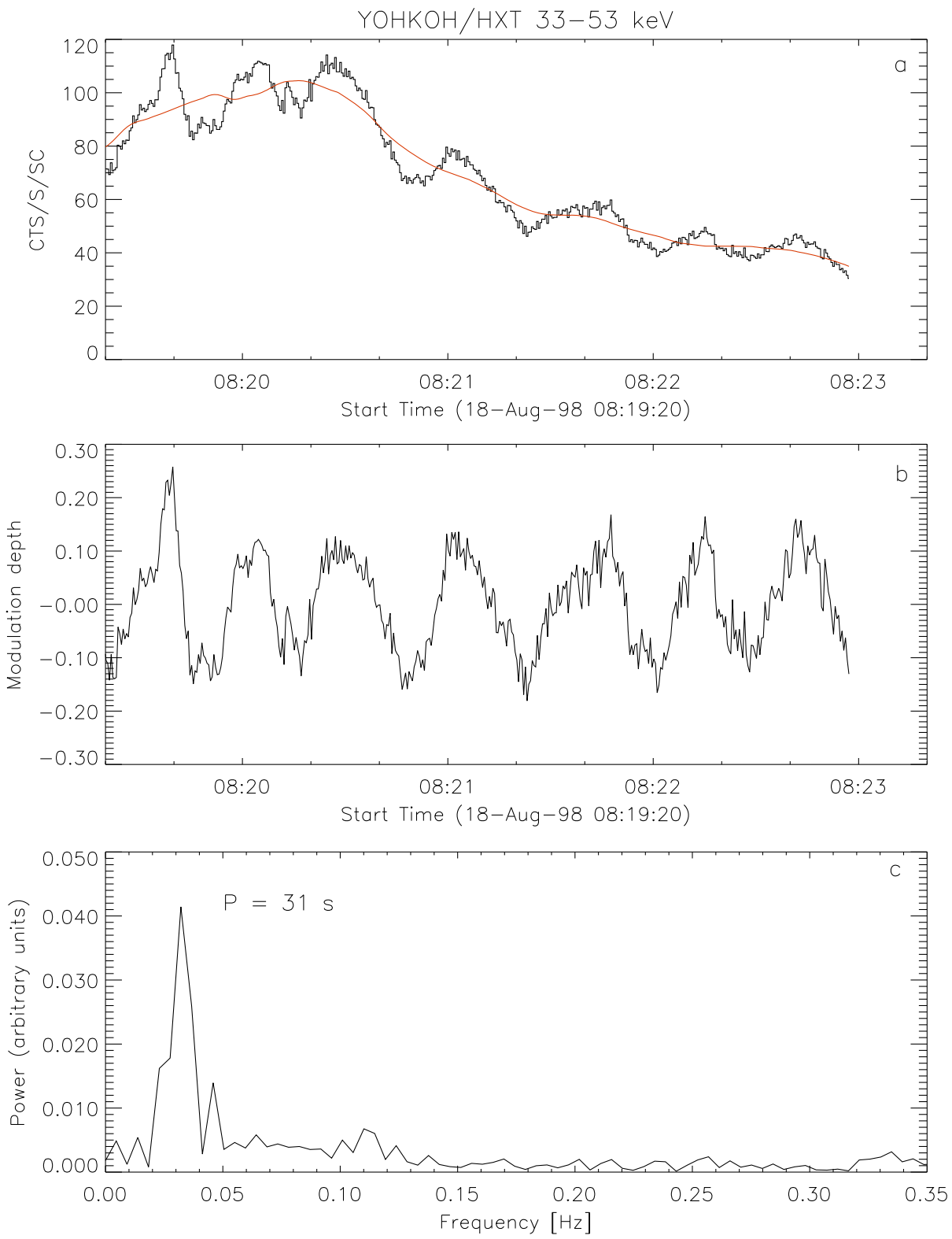

Figure 6 The power-spectrum analysis for the time interval B of the flare on 18 August 1998 (cf. Figure 4).

Next we determined the mean temperature, $T$, emission measure, $E M$, and the mean electron number density, $N$, in the HXR loop-top source, from its Yohkoh soft-X-ray (SXR) images, using the filter-ratio method (from Be119 and Al12 images). This analysis was done in the following way. First we integrated SXR fluxes from the loop-top area $A=4$ Yohkoh pixels [i.e. $4.9 \times 4.9(\operatorname{arcsec})^{2}$ ] at the center of the HXR loop-top source. Next we determined the diameter, $d$, of the HXR source according to the isocontour $0.5 I_{\max }$, where 

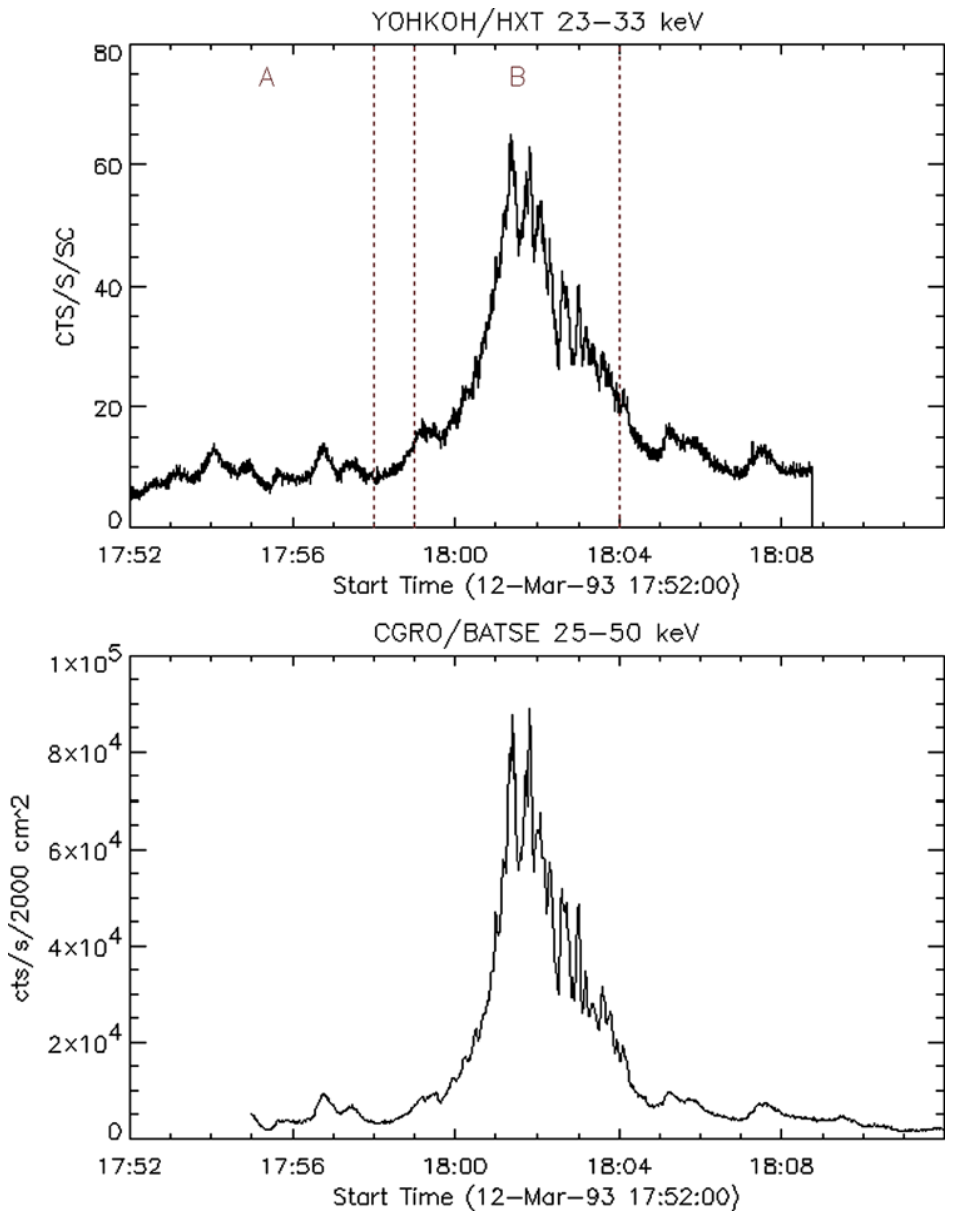

Figure 7 HXR light curves for the flare on 12 March 1993 recorded by Yohkoh/HXT and CGRO/BATSE. Very good agreement between the fluctuations shown in the two curves is seen. The power-spectrum analysis has been carried out for time intervals $\mathrm{A}$ and $\mathrm{B}$, which are marked by vertical lines.

$I_{\max }$ is the maximum intensity within the source. We assumed that the extension of the HXR source along the line of sight is $1.15 \mathrm{~d}$ and calculated the volume of the emitting plasma as $V=1.15 \mathrm{Ad}$ (here 1.15 is a correction factor taking into account that the actual size of the HXR source is somewhat larger than that determined from the isocontour $0.5 I_{\max }$ ); it has been calculated under the assumption that the source is spherical and homogeneous see Figure 1 in Bak-Steślicka and Jakimiec (2005) with $A B / D O=0.5$. The mean electron number density was calculated as $N=\sqrt{E M / V}$ and the obtained time variation of the temperature, $T(t)$, and the density, $N(t)$, are shown in Figures 11 and 12.

Figure 11 shows that the increase of energy release in the loop-top source began already about 23:10:40 UT. The sharp peaks of the temperature about 23:12:30 and 23:16 UT (Figure 11) are correlated with the peaks in HXRs (Figure 3b), which confirms that a significant part of the energy of accelerated electrons is deposited within the loop-top source (the temperature peaks are somewhat delayed, $\sim 16 \mathrm{~s}$, relative to the HXR peaks, which is due to the accumulation of the plasma heating by the accelerated electrons). Significant random 

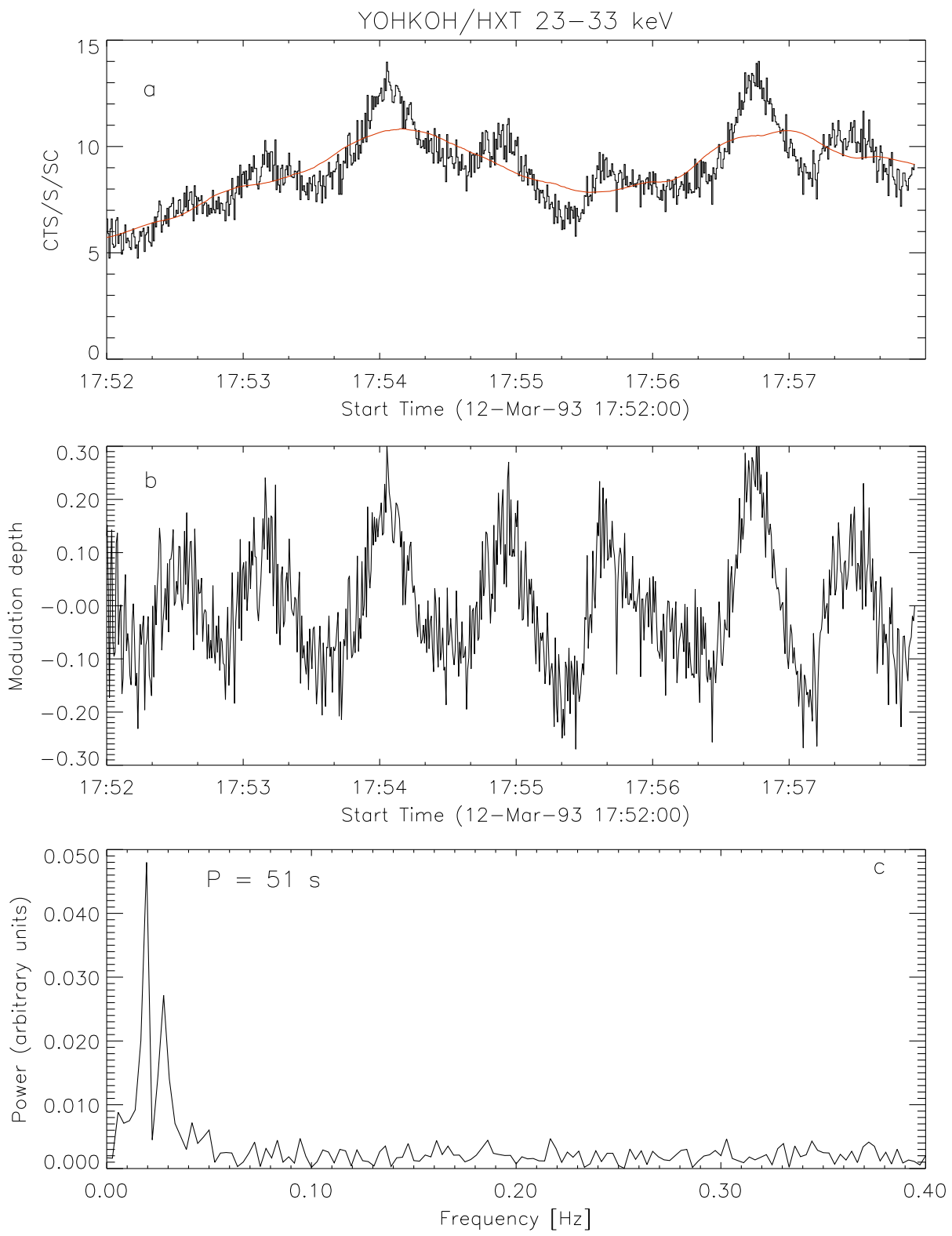

Figure 8 The power-spectrum analysis for the time interval A of the flare of 12 March 1993 (cf. Figure 7).

fluctuations of $N(t)$ in Figure 12 are mostly due to random errors in estimating the source volume from individual HXR images. The systematic increase of the density with time seen in Figure 12 is due to the chromospheric evaporation flow.

X-ray images for the flare of 30 October 1992 are shown in Figure 13. We see similar behavior like in Figure 10. The footpoint sources were stronger than the loop-top source before the impulsive phase and the loop-top source was stronger during the impulsive phase. 

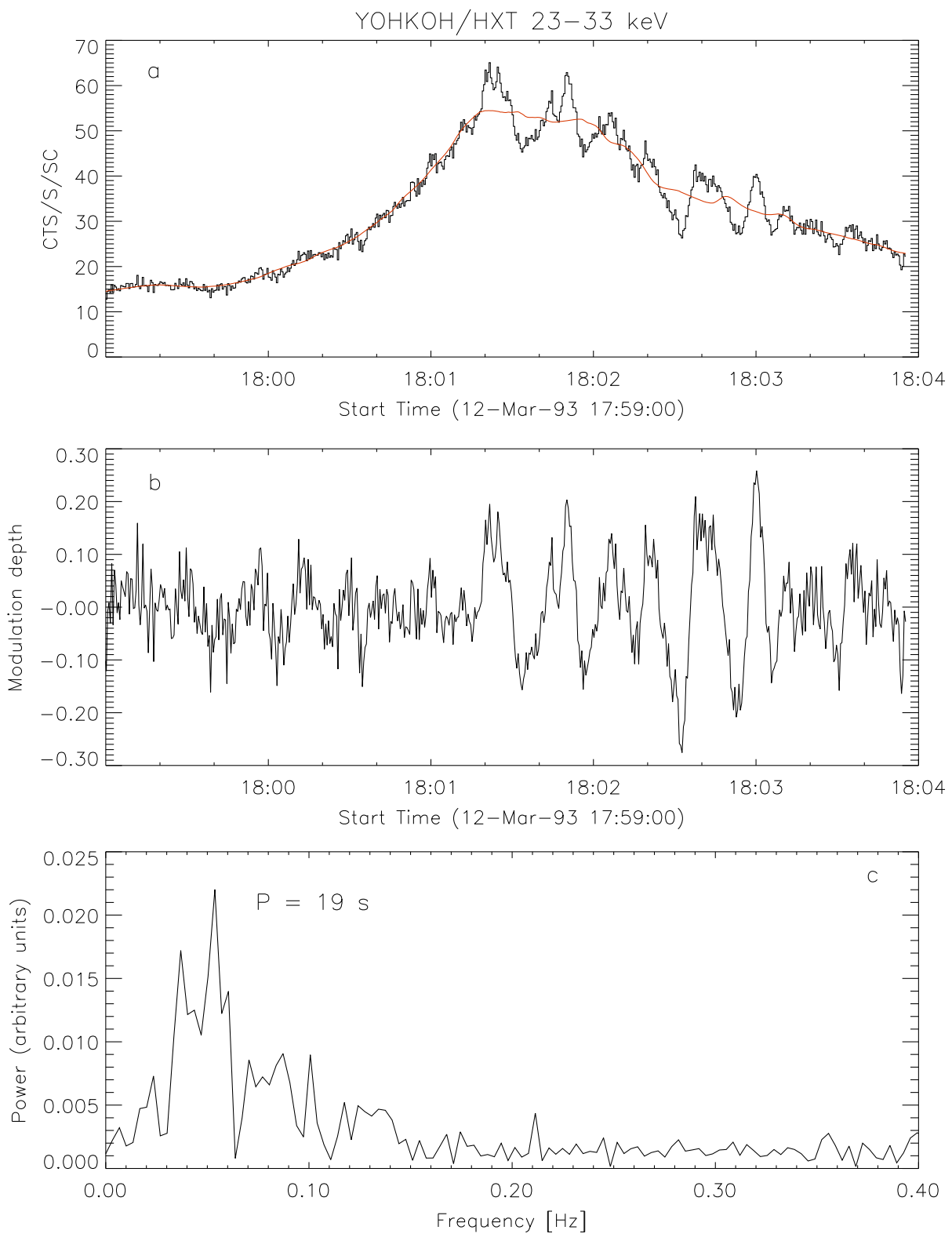

Figure 9 The power-spectrum analysis for the time interval B of the flare of 12 March 1993 ( $c f$. Figure 7).

\subsection{Determination of the Magnetic Field Strength and the Electron Densities for Flares Investigated in Paper I}

In Paper I we used $N=1.0 \times 10^{10} \mathrm{~cm}^{-3}$ as the typical value of the electron number density in the HXR loop-top sources during the impulsive phase. This value was obtained by 

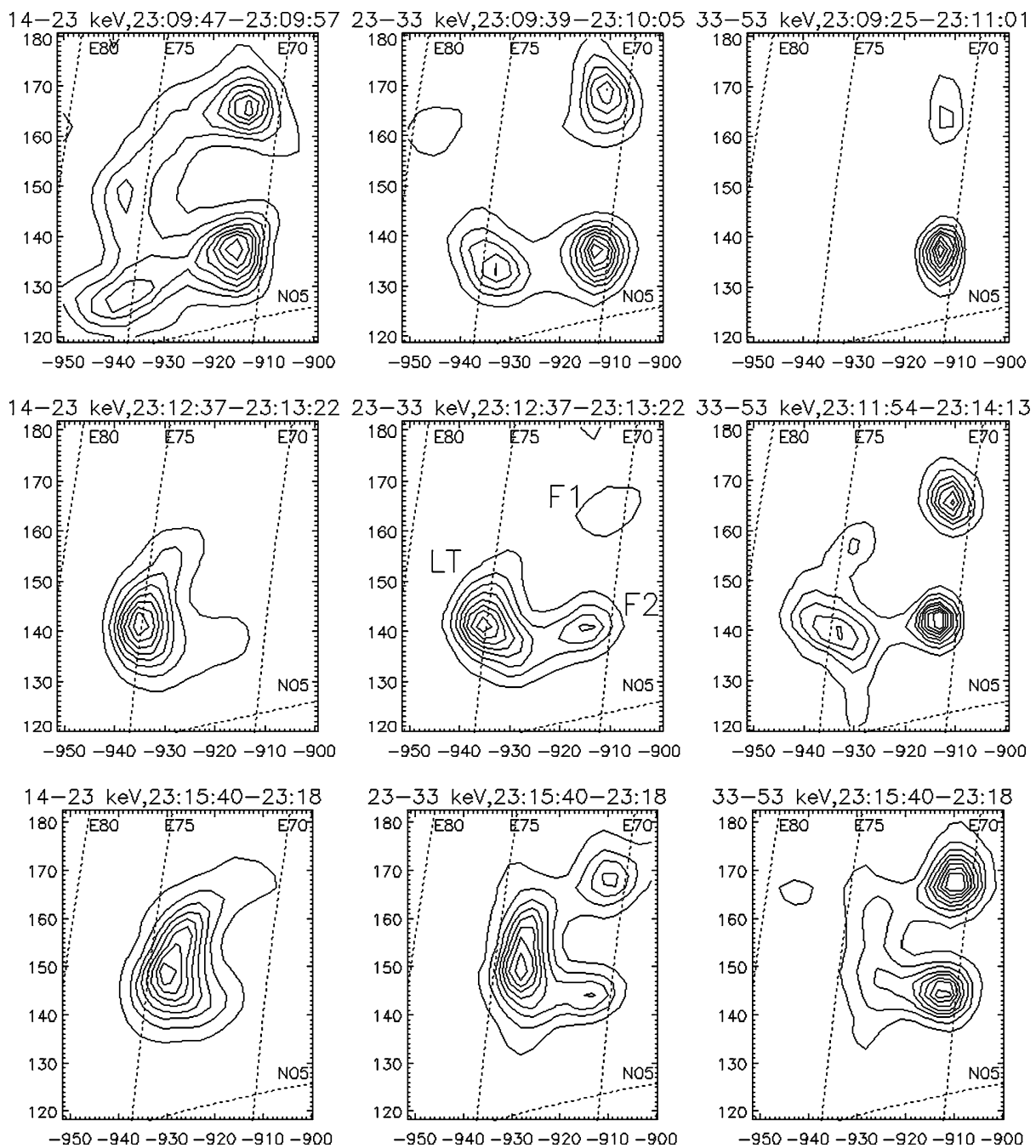

Figure 10 HXR images of the flare of 16 January 1994 in three energy channels: 14-23, 23-33, and $33-53 \mathrm{keV}$ (vertical columns). Upper row: images recorded before the impulsive phase, middle row: recorded at the beginning of the impulsive phase, lower row: recorded during the impulsive phase. See the text for discussion.

Krucker and Lin (2008) from RHESSI soft-X-ray images of the sources. In Paper I we used this value of $N$ to estimate the magnetic field strength in oscillating magnetic traps:

$$
B_{2}=v_{2} \sqrt{4 \pi \rho},
$$

where $v_{2}$ is the wave speed estimated from the analysis of HXR oscillations and $\rho \approx N m_{\mathrm{H}}$, $m_{\mathrm{H}}$ being the mass of proton. The values of $B_{2}$ obtained are given in Table 2 .

For eight flares of those investigated in Paper I it was possible to determine the mean electron number density, $N$, for the HXR loop-top source from the SXR images using the method described in Section 2.2. The values are given in Table 2. We see that most values are significantly higher than those assumed in Paper $\mathrm{I}\left(N=1.0 \times 10^{10} \mathrm{~cm}^{-3}\right)$. This indicates 
Figure 11 Time variation of the mean temperature in the HXR loop-top source of the 16 January 1994 flare.
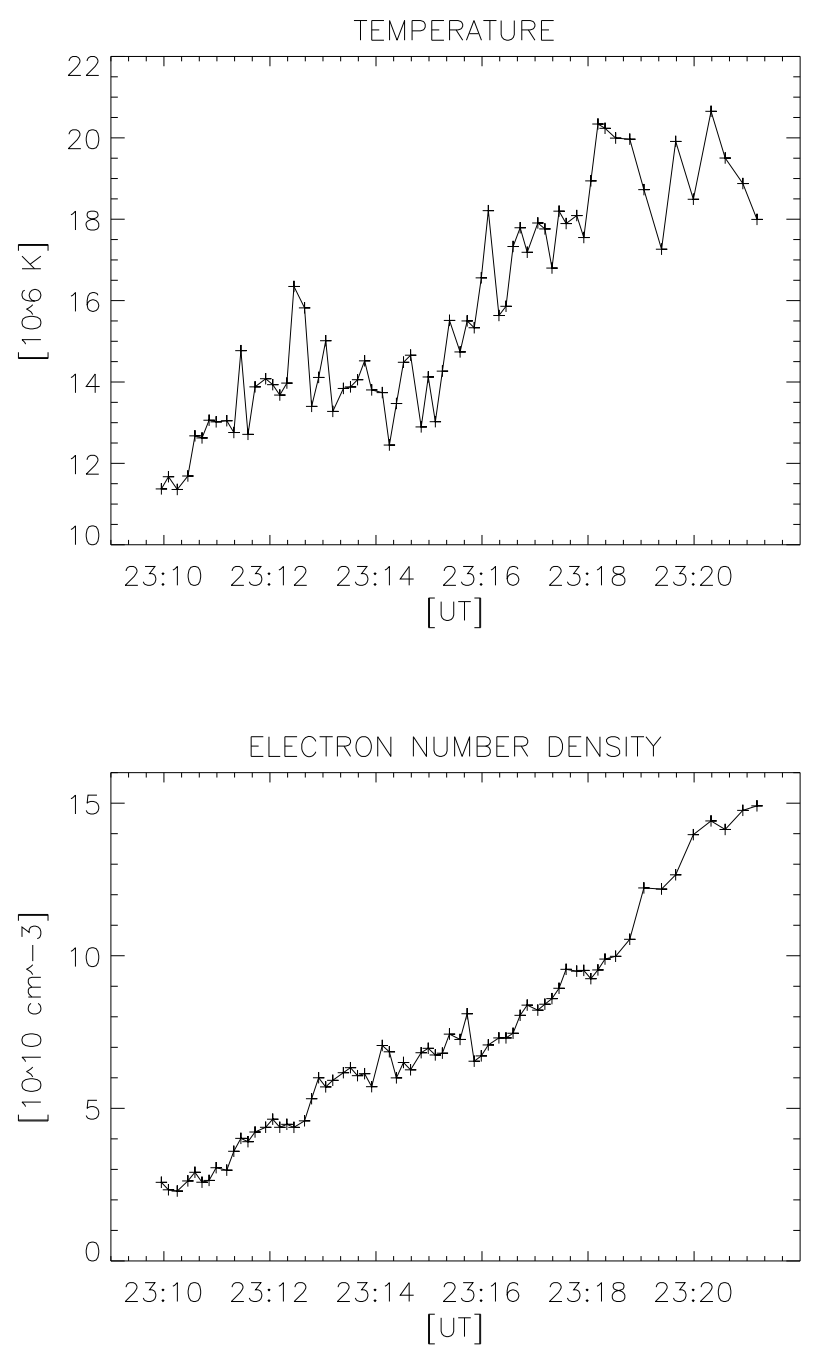

Figure 12 Time variation of the mean electron number density in the HXR loop-top source of the 16 January 1994 flare.

that the value of $N$ used in Paper I, and therefore also the magnetic field strength, $B_{2}$, were underestimated. We have calculated corrected values, $B_{2}^{*}$, of the magnetic field strength using Equation (2) with the new values of $N$ (see Table 2).

Let us note that the values of the electron number density, $N$, derived from Yohkoh/ SXT images are reliable, since:

i) They have been confirmed by an independent method (see Bak-Steślicka and Jakimiec, 2007; Jakimiec and Bak-Steślicka, 2011).

ii) Determination of $N$ from the Yohkoh/Al12 images does not depend significantly on temperature estimates, since the instrumental response function for the Al12 images is nearly constant in a wide range of temperatures (7-40 MK - see curve $f$ in Figure 9 of Tsuneta et al., 1991). 

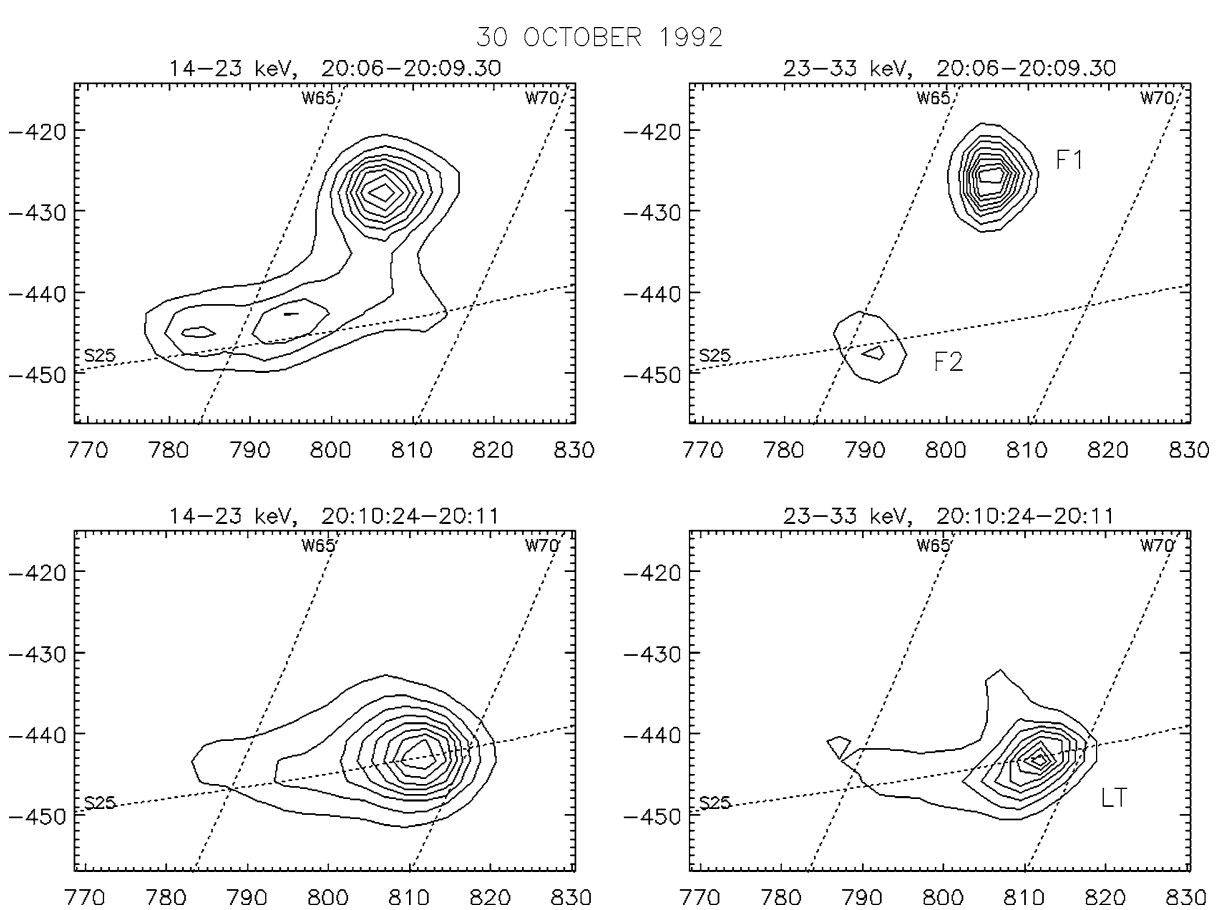

Figure 13 HXR images of the flare of 30 October 1992 in two energy channels: $14-23$ and $23-33 \mathrm{keV}$ (vertical columns). Upper row: images recorded before the impulsive phase, lower row: images recorded during the impulsive phase.

Table 2 Values of mean electron density and magnetic field strength.

\begin{tabular}{lllcr}
\hline Date & $\mathrm{UT}$ & $B_{2}[\mathrm{G}]^{\mathrm{a}}$ & $N\left[10^{10} \mathrm{~cm}^{-3}\right]^{\mathrm{b}}$ & $B_{2}^{*}[\mathrm{G}]^{\mathrm{c}}$ \\
\hline 28 Jun 92 & $14: 00$ & 36 & 1.6 & 45 \\
8 May 98 & $01: 59$ & 40 & 2.3 & 62 \\
31 Oct 91 & $09: 11$ & 26 & 6.0 & 64 \\
14 May 93 & $22: 05$ & 30 & 12 & 104 \\
7 Jun 93 & $14: 22$ & 33 & 15 & 127 \\
16 Jan 94 & $23: 17$ & 35 & 8.0 & 98 \\
23 Jun 00 & $14: 26$ & 28 & 15 & 108 \\
25 Nov 00 & $18: 39$ & 21 & 9 & 63 \\
\hline
\end{tabular}

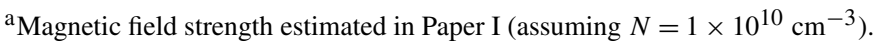

${ }^{b}$ Mean electron number density in HXR loop-top source at HXR maximum, estimated in the present paper.

${ }^{\mathrm{c}}$ Magnetic field strength calculated with the new value of $N$.

\section{Discussion and Summary}

Our explanation of the HXR oscillations is based on two important findings of other researchers: 
Figure 14 (a) Schematic diagram showing a cusp-like magnetic configuration. Magnetic fields $\mathrm{PB}$ and $\mathrm{CP}$ reconnect at $\mathrm{P}$. The thick horizontal line is the chromosphere. (b) Detailed picture of the BPC cusp-like magnetic structure.
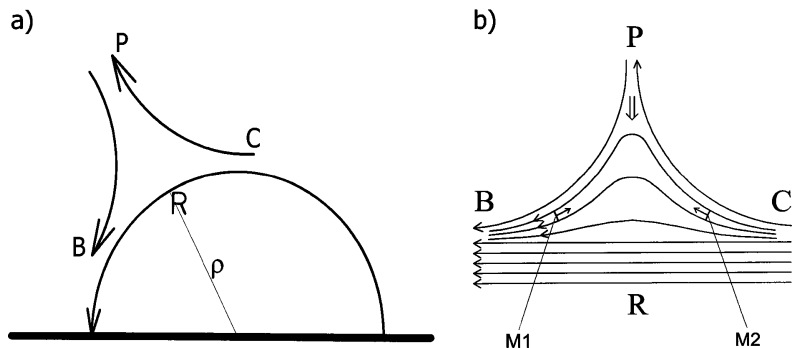

i) The cusp-like (triangular) magnetic structure is commonly accepted as the structure of the magnetic field of a flare (see Aschwanden, 2004a).

ii) Particles are efficiently accelerated during compression (collapse) of magnetic traps (Somov and Kosugi, 1997; Aschwanden, 2004b; Karlický and Kosugi, 2004; Bogachev and Somov, 2005).

A scheme of the cusp-like magnetic structure is shown in Figure 14. Magnetic fields $\mathrm{PB}$ and $\mathrm{PC}$ reconnect at $\mathrm{P}$ (Figure 14a). This generates a sequence of magnetic traps moving downward, the traps overtake each other, they collide and undergo compression (Figure 14b). During the compression particles are accelerated within the traps, magnetic pressure, gas pressure, and the pressure of accelerated particles increase, so that the compression is stopped, the traps can expand and undergo magnetosonic oscillations.

During the compression parameters of the traps undergo strong changes. At the beginning of the compression the trap ratio, $\chi=B_{\max } / B_{\min }$, is high $\left(B_{\max }\right.$ is the magnetic field strength at magnetic mirrors and $B_{\min }$ is the strength at the middle of the trap). The trap ratio decreases during the compression and it reaches the lowest value, $\chi_{\min }$, at the end of the compression. Then the electrons reach the highest energies and they most easily escape from the trap.

The HXR oscillations contain important information about electron acceleration in solar flares:

i) Sharp HXR peaks seen near the HXR maximum (see Figure 1 about 22:04:40 UT), indicate that the electrons are accelerated to the energies $>20 \mathrm{keV}$ very quickly, within a time interval $<0.5 \mathrm{~s}$. They exist in a trap also for a short time: they emit photons (the loop-top emission) and are thermalized within the trap or they quickly escape from the trap and generate the footpoint emission (the ratio between the number of loop-top emitting and escaping electrons is controlled by observations of the ratio between the loop-top and footpoint HXR emission; this ratio is different in different flares, as shown by Tomczak and Ciborski, 2007).

ii) The HXR light curves show that electrons are accelerated continuously. This indicates that in a HXR loop-top source there are many traps whose oscillations are shifted in phase. This is in agreement with the model of excitation of oscillations by a reconnection outflow coming from the reconnection site located at the top of the cusp-like structure (see below).

We consider a single pulse of the magnetic reconnection which generates a pulse of reconnection outflow having a velocity time profile $v(t)$. We describe this time profile as consisting of a central "core" (where $\mathrm{d} v / \mathrm{d} t \approx 0$ ) and "wings" (where $\mathrm{d} v / \mathrm{d} t \neq 0$ ) - like a core and wings in spectral-line profiles. The core of $v(t)$ excites quasi-coherent oscillations of a large volume ("main trap"), which contains many electrons and which is responsible for a sequence of quasi-periodic HXR pulses. 
The wings of the $v(t)$ time profile excite oscillations of many thin ("additional") magnetic traps (see Figure 14b) whose oscillations have different phase shifts due to $\mathrm{d} v / \mathrm{d} t \neq 0$. The superposition of many HXR pulses coming from these additional traps gives a "quasismooth" emission (seen as the emission below the pulses in Figures 1-9). We see that this quasi-smooth emission is stronger than pulses in the investigated flares, which indicates that most of the cusp volume was filled with the "additional" traps (their volume was greater than the volume of the main trap).

Our simple model of oscillating magnetic traps allows us to explain other observed properties of the HXR oscillations. Table 1 shows that for three of our flares (Nos. 2, 3, and 4) the quasi-periodicity, $P$, is significantly less present during the impulsive phase rise. During this time interval the cusp volume is highly crowded with many additional oscillating traps (for two of the flares this is seen in diminished values of Amp $S$ ), which can result in decrease of the length of the main trap (i.e. shift of the magnetic mirrors, M1 and M2 in Figure 14b, toward the trap center).

In many strong flares HXR pulses are not clearly seen during the quick increase of HXR emission, i.e. during the beginning of the impulsive phase (see example in Figure 9). According to our model of oscillating magnetic traps, in such cases the time profile, $v(t)$, of the reconnection flow has broad wings. Therefore, it excites many additional oscillating traps of similar power, which have different lengths (see Figure 14b), and their oscillations are shifted in phase. This gives strong quasi-smooth HXR emission. Clear quasi-periodic pulses are seen only near the maximum of the HXR emission (see Figure 9), when the dominance of the additional traps weakens (see the time variation of Amp $S$ in Table 1).

When we observe a long sequence of quasi-periodic oscillations (like in Figures 1-9), this indicates the following.

i) The oscillations have been excited by a short pulse of the reconnection flow (otherwise the oscillations would be more chaotic).

ii) The oscillations are self-maintained, i.e. some feedback mechanism operates preventing their decay and even leading to their increase during the impulsive phase.

Krucker and Lin (2008) investigated HXR loop-top sources from RHESSI observations and they have found that the energy contained in non-thermal electrons is usually higher than the thermal energy. This means that the pressure of the non-thermal electrons is higher than the gas pressure, $p_{\mathrm{NT}}>p$. This suggests that the pressure, $p_{\mathrm{NT}}$, of non-thermal electrons is an important factor in the feedback mechanism, which maintains the oscillations of magnetic traps. During the compression of a magnetic trap the pressure $p_{\mathrm{NT}}$ steeply increases and this causes an increase of the amplitude of the next expansion of the trap. This, in turn, leads to an increase of the restoring force (i.e. the tension of bent magnetic field lines) and therefore the pulse of the pressure $p_{\mathrm{NT}}$ will be stronger during the next compression. Hence, there is feedback between the intensity of pulses of the pressure $p_{\mathrm{NT}}$ and the amplitude of the magnetic-trap oscillation and this feedback is responsible for the quick increase of the oscillations during the impulsive phase.

It may be interesting to note that this mechanism of maintaining oscillations is analogous to the mechanism of instability that is responsible for stellar pulsations ("non-adiabatic pulsations", see Bradley, 2001): in both cases the point is that additional energy is accumulated during the compression of gas (it is the energy of the helium ionization in the case of Cepheids and it is the energy of non-thermal electrons in our case).

The main results of the present paper are the following:

i) It has been confirmed that quasi-periodic oscillations (QPO) occur in HXR emission of solar flares. 
ii) We have found that low-amplitude QPOs occur before the impulsive phase of some flares.

iii) We have found that the quasi-periodicity $P$ of the oscillations can change in some flares. We interpret this as being due to changes of the length of oscillating magnetic traps.

iv) During the impulsive phase a significant part of the energy of accelerated (non-thermal) electrons is deposited within the HXR loop-top sources (Section 2.2). [For weak HXR flares this is seen at lower energies $(14-23 \mathrm{keV})$, but not at $23-33 \mathrm{keV}$.]

v) We argue that the basic properties of the HXR oscillations can be explained in terms of a simple model of oscillating magnetic traps (see Paper I). This model allows us to explain the large number of electrons accelerated during the impulsive phase: $\mathrm{Ob}-$ servations show that the amplitude of the oscillations quickly increases and therefore the traps are filled with an increasing amount of plasma coming from chromospheric evaporation. This is the main source of the electrons that undergo acceleration.

vi) We suggest that feedback between the pressure of accelerated electrons and the amplitude of the following expansion of a magnetic trap is the mechanism, which causes the quick increase of the amplitude of oscillations.

vii) We have also determined the improved values of the electron number density and magnetic field strength for HXR loop-top sources of several flares investigated in Paper I. The values obtained fall within the limits of $N \approx(2-15) \times 10^{10} \mathrm{~cm}^{-3}, B \approx(45-$ 130) gauss.

Finally, we briefly summarize the development of the ideas concerning electron acceleration within a cusp-like magnetic structure.

In the 1990s it was argued that a fast reconnection jet moving downward generates MHD turbulence and the turbulence accelerates the electrons (see LaRosa and Moore, 1993). However, this idea meets serious difficulties:

i) It is difficult to excite MHD turbulence in the cusp-like volume (LaRosa and Moore, 1993; Frank et al., 1996).

ii) The escape of accelerated electrons from the turbulent volume is difficult (see Jakimiec, 1999) and therefore in this model the HXR loop-top source should always be stronger than footpoint sources, which does not agree with observations.

Somov and Kosugi (1997), Aschwanden (2004b), and Karlický and Kosugi (2004) have worked out a model of electron acceleration in collapsing magnetic traps (first-order Fermi plus betatron acceleration). The advantage of this model is that accelerated electrons can easily escape at the end of trap collapse and they generate strong HXR footpoint emission, in agreement with impulsive phase observations of most flares. But a limitation of this model is that only coronal electrons that are contained in the traps at the beginning of their collapse are accelerated; therefore, the number of accelerated electrons is limited, and not sufficient to explain the recorded HXR emission.

In our Paper I we have combined the above mechanism of electron acceleration, the cusplike magnetic structure and the observations of HXR oscillations to work out the model of electron acceleration in oscillating magnetic traps. The main advantages of this model are the following:

i) During the compression of the traps electrons are accelerated, but during their expansion the traps are filled with dense plasma flowing from below due to chromospheric evaporation; therefore the number of accelerated electrons can be high, in accordance with the HXR observations.

ii) This model has allowed us to indicate the feedback responsible for the quick increase of the HXR pulses and for a quasi-smooth component, i.e. for a quick impulsive-phase rise. 
Acknowledgements The Yohkoh satellite is a project of the Institute of Space and Astronautical Science of Japan. The Compton Gamma Ray Observatory is a project of NASA. We would like to thank anonymous referees for valuable remarks which helped us to improve this paper. This work was supported by the Polish Ministry of Science and High Education, grant No. N N203 193733.

Open Access This article is distributed under the terms of the Creative Commons Attribution License which permits any use, distribution, and reproduction in any medium, provided the original author(s) and the source are credited.

\section{References}

Aschwanden, M.J.: 2004a, Physics of the Solar Corona. An Introduction, Springer, Berlin.

Aschwanden, M.J.: 2004b, Astrophys. J. 608, 554.

Bak-Steślicka, U., Jakimiec, J.: 2005, Solar Phys. 231, 95.

Bak-Steślicka, U., Jakimiec, J.: 2007, Cent. Eur. Astrophys. Bull. 31, 97.

Bogachev, S.A., Somov, B.V.: 2005, Astron. Lett. 31, 537.

Bradley, P.A.: 2001, In: Murdin, P. (ed.) Encyclopedia of Astronomy and Astrophysics, Institute of Physics Publishing, Bristol, article 1862.

Fishman, G.J., Meegan, C.A., Wilson, R.B., Paciesas, W.S., Pendleton, G.N.: 1992, In: Shrader, C.R., Gehrels, N., Dennis, B. (eds.) The Compton Observatory Science Workshop, NASA CP-3137, GSFC, Greenbelt, 26.

Fleishman, G.D., Bastian, T.S., Gary, D.E.: 2008, Astrophys. J. 684, 1433.

Frank, A., Jones, T.W., Ryu, D., Gaalaas, J.B.: 1996, Astrophys. J. 460, 777.

Jakimiec, J.: 1999, In: Wilson, A. (ed.) Magnetic Fields and Solar Processes, vol. 2, SP-448, ESA, Nordwijk, 729.

Jakimiec, J., Bak-Steślicka, U.: 2011, Solar Phys. 272, 91.

Jakimiec, J., Tomczak, M.: 2010, Solar Phys. 261, 233 (Paper I).

Karlický, M., Kosugi, T.: 2004, Astron. Astrophys. 419, 1159.

Kosugi, T., Makishima, K., Murakami, T., Sakao, T., Dotani, T., Inda, M., Kai, K., Masuda, S., Nakajima, H., Ogawara, Y., Sawa, M., Shibasaki, K.: 1991, Solar Phys. 136, 17.

Krucker, S., Lin, R.P.: 2008, Astrophys. J. 673, 1181.

LaRosa, T.N., Moore, R.L.: 1993, Astrophys. J. 418, 912.

Lipa, B.: 1978, Solar Phys. 57, 191.

Melnikov, V.F., Reznikova, V.E., Shibasaki, K., Nakariakov, V.M.: 2005, Astron. Astrophys. 439, 727.

Nakariakov, V.M., Melnikov, V.F.: 2009, Space Sci. Rev. 149, 119.

Somov, B.V., Kosugi, T.: 1997, Astrophys. J. 485, 859.

Tomczak, M., Ciborski, T.: 2007, Astron. Astrophys. 461, 315.

Tsuneta, S., Acton, L., Bruner, M., Lemen, J., Brown, W., Caravalho, R., Catura, R., Freeland, S., Jurcevich, B., Morrison, M., Ogawara, Y., Hirayama, T., Owens, J.: 1991, Solar Phys. 136, 37. 